\title{
Nutrition-Related Cardiovascular Risk Factors in Older People: Results from the Third National Health and Nutrition Examination Survey
}

\author{
Thomas P. Erlinger, MD, MPH, Harold Pollack, MPP, PhD, ${ }^{\dagger}$ \\ and Lawrence J. Appel, MD, MPH*
}

\begin{abstract}
SETTING: In view of the recognized potential benefits of nutritional therapy in older persons, Congress is evaluating the coverage of nutritional services for Medicare beneficiaries.
\end{abstract}

OBJECTIVE: To estimate the number of older persons in the US who have one or more cardiovascular risk factors (hypertension, increased low density lipoprotein (LDL) cholesterol, and diabetes mellitus), for which nutritional therapy is recommended.

DESIGN: Cross-sectional analysis of adults, aged $\geq 65$, participating in the Third National Health and Nutrition Examination Survey (NHANES III).

MAIN OUTCOMES: The authors estimated the proportion of adults, aged $\geq 65$, with diabetes mellitus, increased LDL cholesterol, and/or hypertension. Efforts were made to assess whether obesity status, gender, race, and/or socioeconomic factors were associated with the prevalence of any or all three conditions.

RESULTS: Approximately $86 \%$ (20 million persons) in the US, aged $\geq 65$, have at least one of the index conditions. Whereas a higher body mass index (BMI) increased the likelihood of having any or all three conditions, $81 \%$ of persons of average body weight $\left(\mathrm{BMI}<25 \mathrm{~kg} / \mathrm{m}^{2}\right)$ had at least one condition. After adjusting for age, gender, BMI, marital status, and poverty index, blacks were more likely than whites to have any one condition (odds ratio $(\mathrm{OR})=3.0, P<$ .01 ) or all three conditions ( $O R=2.3, P=.05)$.

CONCLUSIONS: Almost $90 \%$ of Americans aged $\geq 65$ have one or more nutrition-related cardiovascular risk factors. Improved nutritional interventions may be valuable especially for blacks, who have a higher prevalence of conditions requiring nutritional therapy. J Am Geriatr Soc 48:1486-1489, 2000.

Key words: elderly; hypertension; diabetes; hyperlipidemia;

\footnotetext{
From "The Welch Center for Prevention, Epidemiology and Clinical Research, The Johns Hopkins Medical Institutions, Department of Medicine, Baltimore, Maryland; and the University of Michigan School of Public Health, Ann Arbor, Michigan.

This work was supported, in part, by Grant T32PE10025 from the National Institutes of Health.

Address correspondence to Thomas P. Erlinger, MD, MPH, The Welch Center for Prevention, Epidemiology, and Clinical Research, 2024 East Monument St., Suite 2-600, Baltimore, MD 21205.
}

nutrition; Third National Health and Nutrition Examination Survey (NHANES III)

 ardiovascular diseases (CVDs), including stroke and coronary heart disease (CHD), remain a major cause of avoidable mortality and morbidity in the US. Despite declines in age-specific CHD mortality rates over the past two decades, CVDs remain the leading cause of death for Americans over the age of $65 .^{1} \mathrm{~A}$ direct independent association has been demonstrated between nutrition-related risk factors (increased low density lipoprotein (LDL) cholesterol, hypertension, and diabetes), morbidity, and healthcare expenditures in older people. ${ }^{2}$

Dietary advice by physicians and more extensive nutritional therapy by other providers are integral components of the management of these risk factors. ${ }^{3-5}$ For instance, nutrition-based interventions have been shown to improve dietary control and to reduce the need for medication in older persons with diabetes and hypertension. ${ }^{6,7}$

In view of such findings, many professional associations recommend nutritional therapy as initial and adjunctive therapy in the management of hypertension, diabetes, and increased cholesterol. However, only $50 \%$ of people aged $>65$ report receiving any nutritional advice by their physicians to reduce their risk of heart disease or stroke. ${ }^{8}$ Furthermore, contemporary strategies designed to accomplish nutritionrelated lifestyle changes rely heavily on nonphysicians, principally dietitians. Current Medicare policies do not reimburse such nonphysician services provided in the ambulatory setting. However, the US Congress is currently evaluating the appropriateness of extending Medicare coverage to include nutritional guidance by nonphysicians.

To provide information about this policy debate and to define the boundaries of potential Medicare reimbursement, we estimated the burden of nutrition-related cardiovascular risk factors in older adults in the US population, namely, elevated LDL cholesterol levels, hypertension, and diabetes, as well as the co-occurrence of these conditions. We also identified important subgroups within the population that would benefit especially from the provision of nutritional support services.

\section{METHODS}

We analyzed data from the Third National Health and Examination Survey (NHANES III), a nationally representa- 
tive population survey conducted between 1988 and 1994 . NHANES III sampled persons aged $\geq 2$. A total of 30,818 persons were examined including 5252 persons aged $\geq 65$. Of these, 1425 persons aged $\geq 65$ were fasting between 9 and 24 hours at the time of the blood collection and had data on all covariates.

Using probability-sampling weights as well as the strata and primary sampling unit pairings that account for the complex survey design of NHANES III, we estimated the proportion of adults, aged $\geq 65$, in the US with one or more nutrition-related cardiovascular risk factors, e.g., hypertension, elevated LDL cholesterol, and diabetes mellitus. These conditions were chosen because they are common among the older people and because nutritional counseling is recommended routinely as part of their management. LDL cholesterol and not total cholesterol was selected because guidelines for lipid lowering generally target LDL cholesterol and because elevated total cholesterol may also indicate raised high density lipoprotein levels. ${ }^{4}$

Hypertension was defined as having an average systolic blood pressure of $\geq 140 \mathrm{~mm} \mathrm{Hg}$ and/or a diastolic blood pressure of $\geq 90 \mathrm{~mm} \mathrm{Hg}$ and/or taking blood pressurelowering medication. High LDL cholesterol was defined as having an LDL cholesterol of $\geq 130 \mathrm{mg} / \mathrm{dL}$ and/or taking cholesterol-lowering medication. LDL cholesterol was calculated from the Freidwald equation using triglycerides measured after an overnight fast. Therefore, all estimates that utilize LDL cholesterol directly or that depend on LDL cholesterol levels in their definition were performed only on those persons who had fasting blood samples. Estimates of the total prevalence of diabetes and hypertension were obtained from all persons who were interviewed.

Persons were considered to be diabetic if a physician had ever told them that they had diabetes and/or they had a fasting plasma glucose $\geq 126 \mathrm{mg} / \mathrm{dl}$. This methodology is likely to underestimate true diabetes prevalence because it fails to include undiagnosed cases.

Being overweight and obese increase the risk for hypertension, elevated LDL cholesterol, and diabetes. We, therefore, estimated the total population size for each of three weight categories using the most recent National Heart, Lung and Blood Institute definitions: non-overweight (body mass index (BMI) $<25 \mathrm{~kg} / \mathrm{m}^{2}$ ), overweight (BMI $\geq 25-29.9 \mathrm{~kg} /$ $\left.\mathrm{m}^{2}\right)$, and obese $\left(\mathrm{BMI}=30 \mathrm{~kg} / \mathrm{m}^{2}\right) .{ }^{9}$ Estimates of the proportion of individuals with the specified conditions were calculated excluding those observations with missing data on other covariates. To assess the impact of missing values, we performed sensitivity analyses under two opposite conditions: (1) we assumed that all persons with missing values had the specified condition, and (2) we assumed that all persons with missing values did not have the specified condition. Each approach produced similar point estimates.

Descriptive data are expressed as the proportion of individuals with the specified condition(s). Age- and genderadjusted trend tests across BMI categories were calculated using weighted multivariate regression analysis. To determine whether certain factors (obesity status, race, gender, education, marital status, and/or poverty level) were associated with the occurrence of any or all nutrition-related cardiovascular risk factors, we used age-adjusted sample weighted logistic regression. Educational status (high school or more, yes/no), marital status (married, yes/no), and poverty index ratio (PIR) ( 1 vs $>1$ ) were coded as dichotomous variables. The PIR takes into account the total household income and family size of the respondent and is adjusted for inflation, thus allowing it to be used to compare data over time. A PIR $<1.0$ indicates poverty, according to the US Census Bureau standards. STATA software (Release 6, STATA Corp., College Station, TX) was used in all calculations.

\section{RESULTS}

Table 1 displays the crude proportion of adults aged $\geq 65$ with hypertension, elevated LDL cholesterol, and diabetes, alone and in combination, stratified by obesity status. Of an estimated 22.9 million people aged $\geq 65,86 \%$ (20 million) are estimated to have elevated LDL cholesterol, diabetes, and/or hypertension. The prevalence of any condition remained relatively constant across different age groups, 65 to 74 (84\%), 74 to $85(88 \%)$, and $>85(90 \%)$.

Each of the conditions was more likely to occur in combination with either or both of the other two conditions. For instance, $88 \%$ of individuals with diabetes had at least one other condition. Similarly, $68 \%$ of those with high LDL cholesterol and $69 \%$ of those with hypertension had at least one other condition.

The prevalence of diabetes increased across categories of weights, i.e., $9 \%$ among non-overweight, $16.8 \%$ among overweight, and $25.2 \%$ among obese individuals $(P$ trend $<.001$ ). Similar patterns were observed for high LDL cholesterol $(53 \%, 61 \%$, and $69 \% ; P=.003)$ and hypertension $(58 \%, 61 \%$, and $73 \% ; P=.003)$. However, even in those with a non-overweight BMI, $81 \%$ of older persons were estimated to have at least one condition. Increased BMI was also associated with increased co-occurrence of all three conditions $(P=.005)$.

Blacks had a higher prevalence than whites of any one ( $94 \%$ vs $85 \%$ ) or all three conditions $(12.4 \%$ vs $4.1 \%)$. Table 2 displays the odds ratios for having any of the three index conditions in four separate models. After adjusting for age and all of the covariates in each model, blacks and persons with a BMI of 30 were more likely to have at least one condition $(\mathrm{OR}=3.0, P<.01$; and $\mathrm{OR}=3.7, P<.01$, respectively). After multivariate adjustment, blacks were also more likely to have all three conditions together $(\mathrm{OR}=2.3$, $P=.05)$. The overall results were similar to those for any condition, reflecting the high degree of comorbidity among individuals who have any of the three conditions studied.

\section{DISCUSSION}

Nutrition-related CVD risk factors are highly prevalent among older Americans. We estimate that $>20$ million persons aged $\geq 65$ have elevated LDL cholesterol, diabetes, and/or hypertension. Under current best-practice treatment guidelines, these individuals would benefit from nutritional therapy as part of their routine management. ${ }^{3-5}$ Our point estimates likely underestimate the need for nutritional intervention because they do not include undiagnosed diabetes, and because they do not consider other conditions for which nutritional therapy is recommended, such as renal disease.

This study also documents the complexity of nutritionrelated medical conditions in older persons. Most individuals who have diabetes, hypertension, or elevated LDL cholesterol also have at least one other condition. These conditions may be metabolically linked via the insulin resistance syndrome. Because comorbidity increases the risk of CVD, ${ }^{10}$ dietary 
Table 1. Crude Proportion (in percentage) of Adults Aged $>65$ in the US with the Specified Condition(s) Overall and by Category of Weight

Weight Category

\begin{tabular}{|c|c|c|c|c|c|}
\hline & & & \\
\hline Condition(s) & All & $\begin{array}{c}\text { Non- } \\
\text { Overweight } \\
\text { BMI <25 }\end{array}$ & $\begin{array}{l}\text { Overweight } \\
\mathrm{BMI} \geq 25-30\end{array}$ & $\begin{array}{c}\text { Obese } \\
\mathrm{BMI} \geq 30\end{array}$ & $P$ Trend \\
\hline
\end{tabular}

Prevalence of Each Condition According to the Presence or Absence of Other Conditions

One condition

Diabetes only

Hypertension only

High LDL-c only

1.7

21.3

19.8

1.0

25.5

20.8

2.7

20.6

19.9

Two conditions

$\mathrm{DM}+\mathrm{HTN}$

DM + High LDL-C

HTN + High LDL-C

Any of the three conditions

All three conditions
3.5

2.1

1.1

1.1

29.2

86.0

4.5
80.9

2.2

5.4

58.0

53.2
3.2
3.4
32.5
87.4
5.2

14.4

61.4

61.0

$\begin{array}{rl}1.2 & .9 \\ 14.4 & .02 \\ 17.7 & .2\end{array}$

$\begin{array}{rc}8.6 & <.001 \\ 1.5 & .6 \\ 42.1 & <.001 \\ 93.3 & <.001 \\ 7.8 & .007\end{array}$

19.0

72.9

$<.001$

.004

.003

Estimated population sizes in millions are 22.9 (total), 9.3 (BM <25), 8.7 (BMI $\geq 25-29.9$ ), and 4.9 (BMI $\geq 30$ ).

$P$ values are age- and gender-adjusted.

$\mathrm{BMI}=$ body mass index; $\mathrm{LDL}=$ low density lipoprotein; $\mathrm{DM}=$ diabetes mellitus; $\mathrm{HTN}=$ hypertension.

Table 2. Odds Ratios (CI) of Having ANY and ALL of the Index Conditions in Adults Aged $\geq 65$ years in the US

\begin{tabular}{|c|c|c|c|c|}
\hline \multirow[b]{2}{*}{ Condition(s) } & \multicolumn{2}{|c|}{$\begin{array}{l}\text { Odds Ratio of } \\
\text { ANY Condition }\end{array}$} & \multicolumn{2}{|c|}{$\begin{array}{c}\text { Odds Ratio of } \\
\text { ALL } \\
\text { Conditions }\end{array}$} \\
\hline & OR & $95 \% \mathrm{Cl}$ & OR & $95 \% \mathrm{Cl}$ \\
\hline Overweight (BMI 25-30) & $1.7^{\dagger}$ & $(1.0-3.0)$ & $2.4 \dagger$ & $(1.1-5.6)$ \\
\hline Obese $(B M I \geq 30)$ & $3.6^{*}$ & $(1.9-6.9)$ & $3.4^{*}$ & $(1.3-9.8)$ \\
\hline Female & 1.4 & $(0.8-2.5)$ & 1.4 & $(0.6-3.2)$ \\
\hline Black & $2.9^{*}$ & $(1.5-5.5)$ & $2.7^{\dagger}$ & $(1.1-6.3)$ \\
\hline Education ( $\geq$ high school) & 1.1 & $(0.6-1.9)$ & 0.6 & $(0.3-1.5)$ \\
\hline Married & 1.2 & $(0.7-2.2)$ & 1.5 & $(0.6-3.5)$ \\
\hline Poverty index $\leq 1.0$ & 0.8 & $(0.4-1.5)$ & 1.6 & $(0.6-4.1)$ \\
\hline
\end{tabular}

Models are also adjusted for age.

$* P<.01 ;{ }^{\dagger} P<.05$.

modification is especially important in these individuals. Multiple conditions increase the complexity of required behavior change, and may therefore increase the marginal benefits as well as the costs associated with nutritional interventions.

Prior studies ${ }^{11}$ have indicated that overweight patients are more likely than non-overweight individuals to receive physician nutrition and exercise advice. Although our analyses reaffirm the importance of obesity as an individual risk factor, a substantial majority of nonobese older men and women also have one or more nutrition-related conditions. Therefore, BMI status alone is an insensitive marker for the provision of dietary advice and other nutritional services.
This analysis also demonstrates that older blacks are more likely than whites to experience these conditions. This racial disparity is particularly troubling in light of studies documenting that blacks experience greater benefit from nutritional interventions than their white counterparts. In the DASH trial, blacks achieved twice the amount of systolic blood pressure reduction as whites $(6.9 \text { vs } 3.1 \mathrm{~mm} \mathrm{Hg})^{12}$ from a diet rich in fruits, vegetables, and low fat dairy and reduced in total and saturated fat.

Given the high prevalence of these conditions in blacks and the promising results of associated dietary interventions, blacks represent an especially important population for clinical and policy intervention. Several localities funded by the CDC REACH (Racial and Ethnic Approaches to Community Health) 2010 initiative are exploring novel approaches to diabetes and CVD management among blacks. These community-based interventions may provide further guidance to clinicians and policymakers designing nutritional interventions for blacks. ${ }^{13}$

One recent study ${ }^{14}$ suggested that medical nutritional therapy is likely to be cost effective in the older population. Currently, nutritional therapy in the ambulatory setting is not covered by most private insurance policies or by Medicare. Under the proposed Medicare Medical Nutrition Act of 1999 (H.R. 1187/S. 660), Medicare would reimburse "nutritional diagnostic, therapy, and counseling services for the purpose of disease management which are furnished by a registered dietitian or nutrition professional pursuant to a referral by a physician." A recent Institute of Medicine report endorsed the concept of Medicare reimbursement for physician-referred nutrition services in outpatient settings. ${ }^{15}$

Because $86 \%$ of Americans aged $\geq 65$ have at least one nutrition-related CVD risk factor, such legislation might have 
a substantial impact on the health and well-being of Medicare recipients. Were such legislation passed, specific Medicare reimbursement policies would require further research. Current data do not identify the most cost-effective modalities of nutritional intervention. With important exceptions, the relative merits of individual and group nutrition counseling for Medicare recipients have received limited attention in the research literature. Even in the absence of expanded coverage, millions of older Americans would likely benefit from more comprehensive dietary advice by healthcare professionals.

In conclusion, the vast majority of older Americans have at least one CVD risk factor for which required nutritional therapy is recommended. Improved nutritional interventions may be valuable especially for blacks, who have a higher prevalence of conditions that require nutritional therapy.

\section{REFERENCES}

1. Smith BL, Martin JA, Ventura SJ. Births and deaths: Preliminary data for July 1997-June 1998. Natl Vital Stat Rep 1999;47:1-32.

2. Schauffler HH, D'Agostino RB, Kannel WB. Risk for cardiovascular disease in the elderly and associated Medicare costs: The Framingham Study. Am J Prev Med 1993;9:146-154.

3. The sixth report of the Joint National Committee on prevention, detection, evaluation, and treatment of high blood pressure. Arch Intern Med 1997; 157:2413-2446.

4. Summary of the second report of the National Cholesterol Education Program (NCEP) Expert Panel on Detection, Evaluation, and Treatment of High Blood Cholesterol in Adults (Adult Treatment Panel II). JAMA 1993;269: 3015-3023.

5. Franz MJ, Horton ESS, Bantle JP et al. Nutrition principles for the management of diabetes and related complications. Diabetes Care 1994;17:490-518.
6. Collins RW, Anderson JW. Medication cost savings associated with weight loss for obese non-insulin-dependent diabetic men and women. Prev Med 1995;24:369-374.

7. Whelton PK, Appel LJ, Espeland MA et al. Sodium reduction and weight loss in the treatment of hypertension in older persons: A randomized controlled trial of nonpharmacologic interventions in the elderly (TONE). TONE Collaborative Research Group. JAMA 1998;279:839-846.

8. Physician advice and individual behaviors about cardiovascular disease risk reduction: Seven states and Puerto Rico, 1997. MMWR 1999;48:74-77.

9. National Institutes of Health. Clinical Guidelines on the Identification, Evaluation, and Treatment of Overweight and Obesity in Adults: The Evidence Report. NIH Publication No. 98-4083. Bethesda, MD: National Institutes of Health, 1998:1-5.

10. Kannel WB, Neaton JD, Wentworth $D$ et al. Overall and coronary heart discase mortality rates in relation to major risk factors in 325,348 men screened for the MRFIT: Multiple Risk Factor Intervention Trial. Am Heart J 1986;112:825-836.

11. Kreuter MW, Scharff DP, Brennan LK, Lukwago SN. Physician recommendations for diet and physical activity: Which patients get advised to change? Prev Med 1997;26:825-833.

12. Appel LJ, Moore TJ, Obarzanck E et al. A clinical trial of the effects of dietary patterns on blood pressure: DASH Collaborative Research Group. N Eng! J Med 1997;336:1117-1124.

13. Centers for Disease Control and Prevention. CDC awards funding to community coalitions for projects to help eliminate racial and ethnic health disparities. Available at: http://www.cdc.gov/od/oc/media/pressrel/ r990930.html. Accessed September 30, 1999.

14. Sheils JF, Rubin R, Stapleton DC. The estimated costs and savings of medical nutrition therapy: The Medicare population. J Am Diet Assoc 1999;99: 428-435.

15. Institute of Medicine Committee on Nutrition Services for Medicare Beneficiaries. The Role of Nutrition in Maintaining Health in the Nation's Elderly: Evaluating Coverage of Nutrition Services for the Medicare Population. Washington, DC: National Academy Press, 2000. 